\section{(2) OPEN ACCESS}

\title{
Junior doctors and conscientious objection to voluntary assisted dying: ethical complexity in practice
}

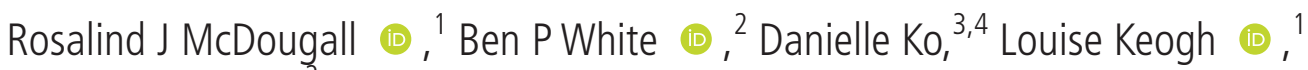 \\ Lindy Willmott
}

${ }^{1}$ Melbourne School of

Population and Global Health, The University of Melbourne, Melbourne, Victoria, Australia ${ }^{2}$ Australian Centre for Health Law Research, Queensland University of Technology, Brisbane, Queensland, Australia ${ }^{3}$ Department of Palliative Care, Austin Health, Heidelberg,

Victoria, Australia

${ }^{4}$ Department of Quality and Patient Safety, Austin Health, Heidelberg, Victoria, Australia

\section{Correspondence to} Dr Rosalind J McDougall, Melbourne School of Population and Global Health, The University of Melbourne, Melbourne, VIC 3010, Australia; rmcdo@unimelb.edu.au

Received 2 December 2020 Revised 12 April 2021 Accepted 16 April 2021

\section{Check for updates}

(c) Author(s) (or their employer(s)) 2021. Re-use permitted under CC BY-NC. No commercial re-use. See rights and permissions. Published by BMJ.

To cite: McDougall RJ, White BP, Ko D, et al. $J$ Med Ethics Epub ahead of print: [please include Day Month Year]. doi:10.1136/ medethics-2020-107125

\section{ABSTRACT}

In jurisdictions where voluntary assisted dying (VAD) is legal, eligibility assessments, prescription and administration of a VAD substance are commonly performed by senior doctors. Junior doctors' involvement is limited to a range of more peripheral aspects of patient care relating to VAD. In the Australian state of Victoria, where VAD has been legal since June 2019, all health professionals have a right under the legislation to conscientiously object to involvement in the VAD process, including provision of information about VAD. While this protection appears categorical and straightforward, conscientious objection to VAD-related care is ethically complex for junior doctors for reasons that are specific to this group of clinicians. For junior doctors wishing to exercise a conscientious objection to VAD, their dependence on their senior colleagues for career progression creates unique risks and burdens. In a context where senior colleagues are supportive of VAD, the junior doctor's subordinate position in the medical hierarchy exposes them to potential significant harms: compromising their moral integrity by participating, or compromising their career progression by objecting. In jurisdictions intending to provide all health professionals with meaningful conscientious objection protection in relation to VAD, strong specific support for junior doctors is needed through local institutional policies and culture.

\section{INTRODUCTION}

Technically, you can opt out of participating in VAD but how will that go down practically as a junior doctor if your consultant is the one co-ordinating things? (Junior doctor) ${ }^{1}$

Voluntary assisted dying (VAD) has been a topic of substantial debate in many countries in recent years, with increasing momentum around legislative change throughout the world. ${ }^{2}$ Debate, and in some cases legislative reform, in most of Australia's six states has been part of this momentum. Since June 2019, VAD has been permitted in the state of Victoria under the Voluntary Assisted Dying Act 2017 for competent terminally ill patients who meet strict criteria, ${ }^{3}$ with 124 deaths in the first year of operation. ${ }^{4}$ Western Australia (WA) passed similar legislation in 2019 and is commencing operation mid-2021. ${ }^{5}$ In the state of Queensland, a parliamentary committee recommended VAD reform, and the Government has now referred the task of drafting legislation to the Queensland Law Reform Commission. ${ }^{6}$ VAD legislation recently passed in Tasmania, and VAD Bills have been tabled in South Australia (in both Houses of Parliament). ${ }^{7}$
Globally, research and policy have not distinguished between the implications for junior and senior doctors. In research and commentary about VAD and medical practitioners in Australia and elsewhere, policymakers' and researchers' emphasis has been on participating doctors, thus tending to focus on senior doctors. ${ }^{89}$ Position statements on VAD from key medical associations within Australia and internationally ${ }^{10-18}$ do not make direct reference to junior doctors. While some empirical research into physicians' views on VAD includes junior doctors as participants in a broader group, ${ }^{19}$ there is a significant gap in both theoretical and empirical research in relation to junior doctors and VAD.

In this paper, we explore theoretically the ethical complexity of conscientious objection for junior doctors in the context of VAD. In the Victorian legislation, all registered health practitioners are covered by a general conscientious objection provision that aims to afford strong protection for clinicians. ${ }^{3}$ The legislation includes a detailed list of things practitioners are able to refuse, with no obligation to refer or to provide information. Under the Victorian law, senior doctors play the main role in VAD: only senior doctors can assess patients' eligibility for VAD and prescribe the VAD medication. However, junior doctors are also involved in caring for patients who request VAD given the team-based nature of healthcare delivery in hospitals, and thus the conscientious objection provision is relevant to junior doctors too.

We argue that while the ability to conscientiously object to being involved in VAD may appear straightforward in legislation, exercising that right is ethically complex for junior doctors in practice because of their specific position of agency. The medical hierarchy, the structure of junior doctors' work and the ethically contentious nature of VAD make navigating conscientious objection an area in which junior doctors are potentially exposed to significant harms. We argue that these specific harms need to be taken into account by those developing policies and guidelines for VAD implementation, particularly within healthcare institutions, to ensure sensitivity to junior doctors' unique position and appropriate support for this group of practitioners.

Throughout the paper, we use the term 'junior doctors' to mean doctors who have completed medical school (ie, are qualified doctors) and are completing further clinical training under supervision while working in hospitals. In Australian public hospitals, junior doctors are the key medical 
staff involved in the face-to-face medical care of a patient, providing daily medical assessments, ordering relevant investigations, discussing treatment options and plans with patients and their families. In contrast, medical specialists, who are ultimately responsible for the care of the patient, usually provide more high-level management and advice, seeing patients more briefly on weekly or biweekly ward rounds supplemented with telephone advice as required. 'Junior doctors' includes interns (first year of professional practice), residents (second and third years of practice), registrars (either trainees who have not yet been accepted into a specialty training programme, or advanced trainees completing a specialty training programme) and fellows (final 1-2 years of training before getting admitted as a fully qualified specialist). Consultants or specialists or senior medical staff refer to doctors who have completed specialty training and qualified to practise independently in their area of specialty, collectively described here as 'senior doctors'.

\section{JUNIOR DOCTORS AND VAD IN VICTORIA}

In Victoria, where VAD has been legally available since June 2019, junior doctors are unable to play any formal role in VAD eligibility assessments, applications for permits, prescribing or administering VAD medications. Under the Victorian legislation, both the doctors involved are required to either hold a fellowship with a specialist medical college or be a vocationally registered general practitioner (s 10(1)), effectively excluding junior doctors. ${ }^{3}$ In addition, at least one of these two doctors must have practised for 5 years after the relevant specialist qualification (s 10(2)). ${ }^{3}$ One of the doctors must also have 'relevant expertise and experience' in the patient's disease (s $10(3)),{ }^{3}$ a requirement that has been interpreted as requiring the doctor to be a medical specialist in that area. ${ }^{20}$

Whether or not junior doctors should be involved in the provision of VAD was explicitly considered by the Ministerial Expert Panel in WA in developing that state's approach, concluding that it 'is not an appropriate responsibility to place on learning or inexperienced practitioners' (p 58). ${ }^{21}$ However, there is some variation internationally in this regard. While legislation in some countries (such as in the Netherlands) does not impose requirements for particular qualifications and experience for participating doctors, other countries do, at least in some circumstances. $^{22} 23$

In Victoria, while eligibility assessments, prescription and administration of a VAD substance are limited to senior doctors, clinical experience in Victorian hospitals suggests that junior doctors are nonetheless involved in a range of other aspects of patient care relating to VAD. Notwithstanding the deliberate intention to limit VAD activities to specialists and vocationally registered general practitioners, junior doctors working in hospitals may be more involved in the day-to-day care of inpatients requesting VAD than the senior doctors who are ultimately responsible.

For example, patients may first raise VAD with junior doctors, given that patients often feel closer to or more familiar with the junior doctors of the treating medical team. Junior doctors may also be asked by patients to provide more specific information regarding VAD and its related processes, asked by their seniors to refer patients to a hospital VAD coordinator or to the statewide VAD navigators, and need to discuss the VAD request with other members of the multidisciplinary team including their supervising specialists. If additional assessments are required, the junior doctor may be asked to facilitate those referrals with relevant phone calls and paperwork.
If VAD administration occurs in the hospital setting, a junior doctor may need to admit a patient for this purpose, prescribe a patient's non-VAD drugs on the medication chart and provide medical care to the patient in the period leading up to VAD administration. Following a VAD death, a junior doctor may need to examine the patient in order to verify the patient's death, complete the patient's discharge summary, fill out the death certificate, provide emotional support to the family and notify the coroner.

The extent to which junior doctors may be involved in the above activities depends on a number of factors. Some Victorian healthcare institutions have appointed their own VAD coordinators with specialised expertise who act as the central point of contact both for patients requesting information and for healthcare teams. Other institutions lack specific staff to coordinate VAD requests, therefore treating teams, including their junior doctors, play a more central role in assisting patients with all aspects of VAD-related care.

\section{A LEGAL RIGHT TO CONSCIENTIOUS OBJECTION}

Conscientious objection clauses are commonly included in laws permitting morally contentious health services, and in Victoria all health professionals have a right under the VAD legislation to conscientiously object to involvement in the VAD process, including provision of information about VAD. Broadly, conscientious objection clauses are designed to protect the moral integrity of doctors, by making it clear that doctors are allowed to decline to provide a particular service, if providing that service would cause them moral distress. ${ }^{24}$ There are many areas of debate in the ethical and legal literature about conscientious objection provisions in health law, including: whether conscientious objection provisions should exist at all, ${ }^{25}{ }^{26}$ what counts as a conscientious objection, ${ }^{27}{ }^{28}$ whether health professionals should be expected to register or report their conscientious objection, ${ }^{29}$ the relationship between institutions and conscientious objection ${ }^{3031}$ and the impact of conscientious objection on equitable access to services. ${ }^{32-34}$

The importance of allowing conscientious objection is central to key professional associations' positions on VAD. The Australian Medical Association (AMA) states:

[i]f governments decide that laws should be changed to allow for the practice of euthanasia and/or physician assisted suicide, the medical profession must be involved in the development of relevant legislation, regulations and guidelines which protect...doctors who do not want to participate. ${ }^{12}$

While recognising 'divergent views within the medical profession', the association's position overall is not supportive of VAD: '[ $\mathrm{t}$ ]he AMA believes that doctors should not be involved in interventions that have as their primary intention the ending of a person's life' ( $p$ 2). ${ }^{12}$ In contrast, the Royal Australasian College of Physicians (RACP) does not put forward an overall position, on the basis of diverse opinion within their membership. They do state that 'physicians should not be forced to act outside their values and beliefs [but] should not disengage from patients holding different values and beliefs without ensuring that arrangements for ongoing care are in place' (p 10). ${ }^{13}$ In their statement, VAD is framed as 'a matter where individual conscience is important' ( $\mathrm{p} 2$ ) and 'a topic which goes to the heart of many physicians' professional identity and beliefs' $(p$ 9). ${ }^{13}$ 
Aligning with this notion that VAD is a matter where a clinician's individual conscience is important, the VAD legislation in Victoria aims to afford strong protection for doctors' choice. Section 7 of the legislation contains a general conscientious objection provision that covers all registered health practitioners, thus including junior doctors. The provision states:

A registered health practitioner who has a conscientious objection to voluntary assisted dying has the right to refuse to do any of the following-(a) to provide information about voluntary assisted dying; (b) to participate in the request and assessment process; (c) to apply for a voluntary assisted dying permit; (d) to supply, prescribe or administer a voluntary assisted dying substance; (e) to be present at the time of administration of a voluntary assisted dying substance; (f) to dispense a prescription for a voluntary assisted dying substance. ${ }^{3}$

Like most legislation, the Act does not define conscientious objection. ${ }^{35}$ It provides a detailed list of things doctors are able to refuse, with no obligation to refer or provide information. The legislation also gives doctors 7 days to refuse a patient's first request to access VAD and to refuse a referral from the coordinating doctor (ss 13, ss 23). ${ }^{3}$ While health professionals are also protected in WA (s 9), ${ }^{36}$ the WA legislation requires a doctor who has a conscientious objection to advise the patient 'immediately' of his or her refusal and 'immediately' give the patient information about VAD (approved by the Chief Executive Officer of the Department of Health) (s 20(2)(a) and (5)). ${ }^{36}$

Elsewhere in the world, VAD legislation varies in relation to conscientious objection. The federal Canadian legislation offers protection to practitioners not participating in medical sssistance in dying (MAiD) by providing that nothing in the law 'compels an individual to provide or assist in providing medical assistance in dying' (c C-46, ss 241.2(9) $)^{37}$ although the words 'conscientious objection' are not expressly used. In Belgium, the legislation states that no doctor can be compelled to perform euthanasia or assist in performing euthanasia, but places some obligations on the doctor who refuses to do so: the doctor must inform the patient in a timely way, explain the reasons behind his/her refusal and, if requested to do so by the patient, refer the patient to another doctor nominated by the patient (Art 14). ${ }^{38}$ In the Netherlands, the legislation is silent on conscientious objection. The issue is addressed instead by government policy which provides that doctors are not required to grant a request for euthanasia, but their refusal should be discussed with the patient and the doctor may consider referring the patient to another doctor. ${ }^{39}$

\section{ETHICAL COMPLEXITY FOR JUNIOR DOCTORS IN PRACTICE}

While the conscientious objection protection in Victorian law appears categorical and straightforward, conscientious objection to VAD-related care is ethically complex in practice for junior doctors. Their specific place in the medical hierarchy exposes them to potential harms. This is particularly the case for junior doctors wishing to exercise a conscientious objection to VAD when their senior colleagues are participating, but also challenging for junior doctors who want to learn about VAD-related care when their supervisor has a conscientious objection or employing institution has elected not to participate in VAD. A multisite study of Victorian clinicians' views on VAD legislation indicated differences between levels of support for VAD among junior doctors (61\%) compared with senior medical staff (51\%), suggesting that divergent views between junior and senior staff within a team may be common. ${ }^{40}$
Box 1 A case of a junior doctor with a conscientious objection to VAD

Jim is a resident working in oncology. Jim has a conscientious objection to voluntary assisted dying (VAD). Participating in any act that could be seen as facilitating a VAD request is contrary to his deeply held values. This includes participating in conversations involving VAD, advising a colleague of a VAD request or passing a VAD request on to a senior doctor or VAD coordinator. Jim is reviewing Mary, a 65 -year-old woman with advanced pancreatic cancer. During his review, Mary says, 'I can't go on much longer. I have been thinking about assisted dying. Can you tell me about it, and what I need to do to get this process started?' Jim is worried that if he raises his conscientious objection, and declines to pass on Mary's request or provide any additional information, the patient will make a complaint and his team will find out he is a conscientious objector. Jim needs a reference from his consultant as part of his application for a position in the oncology training programme and is worried this will compromise his relationship with his consultant, whose view on VAD is unknown. He also fears being judged as 'not a team player' or 'not patient-centred' by other members of his team. He is very concerned about creating additional work for his already stretched bosses. What should he do?

From an ethical perspective, junior doctors are in a different position of agency compared with their senior colleagues. Their agency is reduced, given that they work at the bottom of the medical hierarchy, under the supervision and direction of their more senior colleagues. They are expected to fulfil their role in the team, implementing the treatment plans led by their consultants. Unlike doctors who have completed their training, junior doctors are simultaneously responsible health professionals and subjugate learners within their team. ${ }^{41}$ Empirical studies report the practical difficulty that junior doctors have in speaking up or acting in line with their values, given this complex position of agency. ${ }^{42} 43$

Further, junior doctors are dependent on their senior colleagues for training opportunities and career progression. They are reliant on senior doctors for references that will determine acceptance into highly competitive specialist training programmes, sought-after training positions and overall career advancement. Of the constraints on the agency of junior doctors due to their position in the medical hierarchy, Parker notes that ' $[t]$ he gradient of the hierarchy alone makes it difficult for them to refuse [seniors' requests] and their reliance on seniors for support, mentorship and education intensifies this'. ${ }^{44}$

Using the conscientious objection provision in practice is not straightforward for junior doctors given their place in the hierarchy. For this group of clinicians, there are substantial social barriers to exercising a conscientious objection, despite the clear statement in the legislation that they have the right to refuse specific tasks associated with VAD. As junior doctors, they could be expected to have a conversation or carry out medical tasks in support of VAD that will cause moral distress, and lack a viable pathway for claiming a conscientious objection without substantial harm. Jim's case in box 1 describes a hypothetical example of this type of situation.

The team-based nature of care and junior doctors' lack of control over their own specific work placements mean that a doctor like Jim cannot necessarily simply avoid working in affected areas. While some institutions might be both large and 
flexible enough to facilitate junior doctors avoiding areas in which VAD requests are more likely, this will not always be possible. Medical cultures also vary between different hospitals. In some hospitals and teams, open discussion is possible; if Jim were in such a hospital, he might feel sufficiently safe to raise his concerns and a positive, productive discussion could follow, alongside high-quality patient-centred care for Mary. The published positions of the AMA and RACP outlined earlier potentially contribute to a medical culture in which conscientious objection and diversity of opinion are acknowledged and accepted. However, anecdotal evidence suggests that in some hospitals, such open discussion between junior and senior medical staff is not encouraged, particularly on ethically or clinically contentious issues. Where this is the case, the pressure for a junior doctor to unquestioningly engage in the work typically seen as their remit as the junior will be stronger. In the context of this expectation, conscientious objection to VAD as a junior doctor becomes a difficult option to enact. Junior doctors' ability to use the legislation's conscientious objection provision effectively is limited by their specific position of agency.

A further complexity for junior doctors is the limited nature of the tasks specified in the conscientious objection provision in the Victorian legislation. Some of the VAD-related tasks which a junior doctor may be asked to perform are not clearly covered by the listed tasks which practitioners have a legal right to refuse. For junior doctors, there are particular role-specific tasks which implicate them in VAD such that they might wish to conscientiously object but their legal right to do so is unclear because the legislation does not specifically list these tasks. ${ }^{i}$ These are tasks such as admitting a patient for VAD administration in the hospital, providing medical care in the lead up to VAD administration, verifying the death, completing the discharge summary and notifying the coroner of a VAD death. A junior doctor wanting to refuse these tasks would be reliant on social and organisational recognition of a more general entitlement of health professionals to conscientiously object to participating in procedures and tasks which conflict with their values, lacking the categorical specific legal right associated with the VAD tasks listed in the legislation.

The concerns outlined focus on junior doctors who have a conscientious objection to VAD. In addition, the conscientious objection provision in Victoria's VAD legislation can also create a different type of difficulty for junior doctors. A junior doctor who is interested in VAD may be unable to develop skills in this area of practice if their consultant has a conscientious objection or their organisation discourages or prohibits VAD. Organisations can range from supportive of patients requesting $\mathrm{VAD}$, to discouraging or even prohibiting VAD. The overall culture and approach to VAD in their local working environment is likely to impact significantly on junior doctors' experience with VAD.

\section{CONCLUSION}

There is an ethically important tension between the broad conscientious objection provision in Victoria's VAD legislation and the practical possibilities for junior doctors within existing health service structures. Junior doctors have particular ethical considerations that limit their agency and, in particular, make

${ }^{\mathrm{i}}$ We are grateful to an anonymous JME reviewer for highlighting this point. exercising a conscientious objection to VAD difficult despite strong legislative protection. Legislative protection alone is insufficient to protect junior doctors who have a conscientious objection to VAD, given that the costs of acting in accordance with their conscience may be substantial. While senior doctors may also face challenges in conscientiously objecting (in relation to workplace conflict, and imposing workload on colleagues), junior doctors' dependence on their senior colleagues for training and career progression gives us reason to be particularly concerned about the situation of the junior doctor. Their subordinate position exposes them to potential significant harms: compromising their moral integrity by participating, or sacrificing career progression by objecting.

VAD laws do not purport to specifically regulate junior doctors' involvement in VAD. This is significant because while the eligibility assessment, oversight of the VAD process and provision of VAD are critical functions outlined in the law, and assigned to senior doctors in some jurisdictions, junior doctors will continue to be involved in VAD in a range of other ways, for example, in conversations about VAD or supporting senior doctors in these roles. This (perhaps understandable) legislative silence about the role of junior doctors means their role is likely to be regulated by other less formal means such as policy, position statements or guidelines. Their work will also be more susceptible to less tangible factors such as the culture at a particular institution regarding expectations of a junior doctor's role in VAD discussions and the care provided to patients who will be receiving VAD in a hospital setting.

This crucial role of less formal mechanisms in shaping junior doctors' VAD role and work points to many areas for future theoretical and empirical investigation. Increasing theoretical insight is needed into the kinds of activities to which a doctor can justifiably object on the basis of conscience; what is the relationship between junior doctors' more peripheral involvement in VAD and moral integrity? Further, understanding how junior doctors could increase their own agency and the nature of ideal institutional support would be fruitful areas for empirical bioethics research with junior doctors, both in the Victorian context and in other jurisdictions where VAD is available.

In jurisdictions like Victoria that aim to provide all health professionals with conscientious objection protection in relation to VAD, specific attention is needed to junior doctors' situation. Unless there is also a strong organisational culture in place to support all staff to act within their moral conscience with clear messaging from hospital leadership, conscientious objection will be difficult for junior doctors in practice. Deliberate and specific assistance for junior doctors is needed through local institutional policies and culture. Junior doctors need to be properly supported to respectfully decline having VAD-related conversations with patients if these conversations would cause moral distress. This can be achieved by strategies such as provision of scripts to junior doctors for these situations and clear messaging from hospital leadership that junior doctors' decisions about their participation will be respected, without disadvantage. Alongside these strategies, organisations need an appropriate escalation pathway to ensure that the patient's request for information or access to VAD is heard and managed appropriately without delay due to conscientious objection. Ideally, these supports need to be in place before the start date for VAD, as many challenges arise in the early stage of implementation as the health system grapples with this change in law.

Acknowledgements We are grateful to Isabella Holmes for her research assistance, specifically sourcing and reviewing literature. 
Contributors All authors conceptualised the paper, wrote sections of the first draft and made substantive revisions throughout the paper. RJM finalised the text, which was approved by all authors.

Funding The project was funded by the Research Grant Support Scheme, Faculty of Medicine, Dentistry and Health Sciences at The University of Melbourne. BPW is a recipient of an Australian Research Council Future Fellowship (project number FT190100410: Enhancing end-of-life decision-making: optimal regulation of voluntary assisted dying) funded by the Australian Government.

Competing interests BPW and LW were engaged by the Victorian Government to design and provide the legislatively mandated training for doctors involved in voluntary assisted dying. DK is a member of the Victorian Voluntary Assisted Dying Review Board. She has engaged in this research in her role as a clinical ethics researcher and palliative care specialist and not in her role as a member of the Board. Any views expressed in this paper are not to be attributed to the Board.

Patient consent for publication Not required.

Provenance and peer review Not commissioned; externally peer reviewed. Data availability statement There are no data in this work.

Open access This is an open access article distributed in accordance with the Creative Commons Attribution Non Commercial (CC BY-NC 4.0) license, which permits others to distribute, remix, adapt, build upon this work non-commercially, and license their derivative works on different terms, provided the original work is properly cited, appropriate credit is given, any changes made indicated, and the use is non-commercial. See: http://creativecommons.org/licenses/by-nc/4.0/.

\section{ORCID iDs}

Rosalind J McDougall http://orcid.org/0000-0002-3809-2575

Ben P White http://orcid.org/0000-0003-3365-939X

Louise Keogh http://orcid.org/0000-0003-2963-6451

Lindy Willmott http://orcid.org/0000-0002-9750-287X

\section{REFERENCES}

1 McDougall R, Hayes B, Sellars M, et al. 'This is uncharted water for all of us': challenges anticipated by hospital clinicians when voluntary assisted dying becomes legal in Victoria. Aust Health Rev 2020;44(3):399-404.

2 White B, Willmott L. Future of assisted dying reform in Australia. Aust Health Rev 2018;42(6):616-20.

3 Victorian Parliament. Voluntary assisted dying act 2017

4 Voluntary Assisted Dying Review Board. Report of operations: January-June 2020. Available: https://www.bettersafercare.vic.gov.au/sites/default/files/2020-08/VADRB_ Report\%20of\%20operations\%20August\%202020\%20FINAL_0.pdf [Accessed Nov 2020].

5 Government of Western Australia, Department of Health. Voluntary assisted dying, 2020. Available: https://ww2.health.wa.gov.au/voluntaryassisteddying\#: :text=0n\% 2010\%20December\%202019\%20the, is\%20scheduled\%20for\%20mid\%2D2021 [Accessed Nov 2020].

6 Hinchliffe S. Terms of Reference: Queensland's laws relating to voluntary assisted dying, 2020. Available: https://www.qlrc.qld.gov.au/_data/assets/pdf_file/0004/ 651379/vad-tor.pdf [Accessed Nov 2020].

7 End-Of-Life choices (voluntary assisted dying) bill has been passed by the Tasmanian legislative assembly and is awaiting passage through the legislative council at time of writing; Voluntary assisted dying bill 2020 (SA).

8 Rutherford J. Conscientious participants and the ethical dimensions of physician support for legalised voluntary assisted dying. J Med Ethics 2020. doi:10.1136/ medethics-2020-106702. [Epub ahead of print: 12 Nov 2020].

9 Evenblij K, Pasman HRW, van Delden JJM, et al. Physicians' experiences with euthanasia: a cross-sectional survey amongst a random sample of Dutch physicians to explore their concerns, feelings and pressure. BMC Fam Pract 2019;20(1):177.

10 Palliative Care Australia. Voluntary assisted dying in Australia: guiding principles for those providing care to people living with a life-limiting illness, 2019. Available: https://palliativecare.org.au/wp-content/uploads/dlm_uploads/2019/06/PCA-GuidingPrinciples-Voluntary-Assisted-Dying.pdf [Accessed Nov 2020].

11 ANZSPM. Australian and New Zealand Society of palliative medicine position statement: the practice of euthanasia and physician-assisted suicide, 2020. Available: https://www.anzspm.org.au/c/anzspm?a=sendfile\&ft=p\&fid=1491523669\&sid= [Accessed Nov 2020].

12 Australian Medical Association. Euthanasia and physician assisted suicide, 2016 Available: https://ama.com.au/position-statement/euthanasia-and-physician-assistedsuicide-2016 [Accessed Nov 2020].

13 The Royal Australasian College of Physicians. Statement on voluntary assisted dying, 2018. Available: https://www.racp.edu.au/docs/default-source/advocacy-library/ racp-voluntary-assisted-dying-statement-november-2018.pdf?sfvrsn=761d121a_4 [Accessed Nov 2020].

14 British Medical Association. The BMA's position on physician-assisted dying, 2020. Available: https://www.bma.org.uk/advice-and-support/ethics/end-of-life/the-bmas- position-on-physician-assisted-dying\#: :text=In\%20full\%2C\%20our\%20policy\% 20states,made\%20legal\%20in\%20the\%20UK [Accessed Nov 2020].

15 Royal College of Physicians. The RCP clarifies its position on assisted dying, 2019. Available: https://www.rcplondon.ac.uk/news/rcp-clarifies-its-position-assisted-dying [Accessed Nov 2020].

16 Association for Palliative Medicine of Great Britain and Ireland. The Association for Palliative Medicine (APM) web materials on actively and intentionally ending life (variously called 'assisted suicide', 'assisted dying', 'aid in dying' and 'euthanasia'), 2020. Available: https://apmonline.org/news-events/apm-physician-assisted-dyingweb-materials/ [Accessed Nov 2020].

17 World Medical Association. WMA Declaration on euthanasia and physician assisted suicide, 2019. Available: https://www.wma.net/policies-post/declaration-oneuthanasia-and-physician-assisted-suicide/ [Accessed Nov 2020].

18 Royal Australian College of General Practitioners. RACGP position statement on voluntary assisted dying legislation, 2019. Available: https://www.racgp.org.au/ advocacy/position-statements/view-all-position-statements/clinical-and-practicemanagement/voluntary-assisted-dying-legislation [Accessed Nov 2020].

19 Kitching M, Stevens AJ, Forman L. Views regarding physician-assisted suicide: a study of medical professionals at various points in their training. Clin Ethics 2008:3(1):27-33.

20 Victoria State Government, Department of Health and Human Services. Voluntary assisted dying - guidance for health practitioners, 2019. Available: https://www2 health.vic.gov.au/about/publications/policiesandguidelines/voluntary-assisted-dyingguidance-for-health-practitioners [Accessed Nov 2020].

21 Government of Western Australia, Department of Health. Expert panel on voluntary assisted dying: final report, 2019. Available: https://ww2.health.wa.gov. au/Articles/U_Z/Voluntary-assisted-dying-ministerial-expert-panel [Accessed Nov 2020].

22 Oregon Health Authority. Oregon Revised Statute: Oregon's Death with Dignity Act 127.800 s.1.01, 1994. Available: https://www.oregon.gov/oha/ph/providerpartnerr esources/evaluationresearch/deathwithdignityact/pages/ors.aspx [Accessed Nov 2020].

23 Belgium. The Belgian act on euthanasia of May, 28th 2002. Eur J Health Law 2003;10(3):329-35 https://apmonline.org/wp-content/uploads/2019/01/belgium-acton-euthanasia.pdf

24 Brock DW. Conscientious refusal by physicians and pharmacists: who is obligated to do what, and why? Theor Med Bioeth 2008;29(3):187-200.

25 Wicclair M. Conscientious objection in healthcare and moral integrity. Camb Q Healthc Ethics 2017;26(1):7-17.

26 Savulescu J, Schüklenk U. Doctors have no right to refuse medical assistance in dying, abortion or contraception. Bioethics 2017:31(3):162-70.

27 Ben-Moshe N. The truth behind conscientious objection in medicine. J Med Ethics 2019;45(6):404-10.

28 Emmerich N. Conscientious objection should not be equated with moral objection: a response to Ben-Moshe. J Med Ethics 2019;45(10):673-4.

29 Minerva F. Professional duties of conscientious objectors. J Med Ethics 2019;45(10):675-6.

30 Shadd $\mathrm{P}$, Shadd J. Institutional non-participation in assisted dying: changing the conversation. Bioethics 2019:33(1):207-14.

31 Sumner LW. Institutional refusal to offer assisted dying: a response to Shadd and Shadd. Bioethics 2019:33(8):970-2.

32 Minerva F. Conscientious objection in Italy. J Med Ethics 2015;41(2):170-3.

33 Keogh L, Croy S, Newton D, et al. General practitioner knowledge and practice in relation to unintended pregnancy in the Grampians region of Victoria, Australia. Rural Remote Health 2019;19(4):5156.

34 McDougall R, Pratt B. Too much safety? Safeguards and equal access in the context of voluntary assisted dying legislation. BMC Med Ethics 2020;21(1):38.

35 Haining CM, Keogh LA, Gillam LH. Understanding the reasons behind healthcare providers' conscientious objection to voluntary assisted dying in Victoria, Australia. J Bioeth Inq 2021;45(5). doi:10.1007/s11673-021-10096-1. [Epub ahead of print: 26 Feb 2021].

36 Western Australian Parliament. Voluntary assisted dying act 2019

37 Government of Canada. Offenses against the person and reputation (cont.), Medical assistance in dying (cont.) Criminal Code RSC 1985, c C-46, ss 241-2(9). Available: https://laws-lois.justice.gc.ca/eng/acts/c-46/page-54.html [Accessed Nov 2020].

38 The Belgian act on euthanasia of May, 28th 2002. Eur J Health Law 2003;10(3):329-35.

39 Government of the Netherlands, Ministry of Health, Welfare and Sport. Is euthanasia allowed? Available: https://www.government.nl/topics/euthanasia/is-euthanasiaallowed [Accessed Nov 2020].

40 Sellars M, Tacey M, McDougall R. Support for and willingness to be involved in voluntary assisted dying: a multisite, cross-sectional survey study of clinicians in Victoria, Australia. Intern Med J. In press

41 McDougall R. The junior doctor as ethically unique. J Med Ethics 2008;34(4):268-70.

42 McDougall RJ. Being 'one cog in a bigger machine': a qualitative study investigating ethical challenges perceived by junior doctors. Clin Ethics 2009;4(2):85-90.

43 Rosenbaum JR, Bradley EH, Holmboe ES, et al. Sources of ethical conflict in medical housestaff training: a qualitative study. Am J Med 2004;116(6):402-7.

44 Parker J. Junior doctors and moral exploitation. J Med Ethics 2019;45(9):571-4. 


\section{University Library}

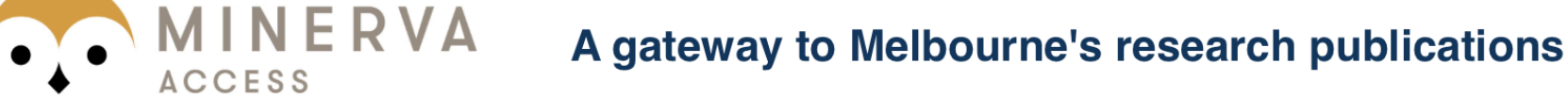

Minerva Access is the Institutional Repository of The University of Melbourne

Author/s:

McDougall, RJ;White, BP;Ko, D;Keogh, L;Willmott, L

Title:

Junior doctors and conscientious objection to voluntary assisted dying: ethical complexity in practice

Date:

2021-06-14

Citation:

McDougall, R. J., White, B. P., Ko, D., Keogh, L. \& Willmott, L. (2021). Junior doctors and conscientious objection to voluntary assisted dying: ethical complexity in practice. JOURNAL OF MEDICAL ETHICS, 48 (8), pp.517-521. https://doi.org/10.1136/ medethics-2020-107125.

Persistent Link:

http://hdl.handle.net/11343/281084

License:

CC BY-NC 